\title{
Impact of Load Modeling in Distribution State Estimation
}

\author{
M. Karimi, Student Member IEEE, H. Mokhlis, Member IEEE, A. H. A. Bakar, Member IEEE, \\ A. Shahriari, Student Member IEEE, M. A. Faradonbeh, Student Member IEEE, H. M. Rosli
}

\begin{abstract}
System state monitoring is crucial in order to maintain the system security, reliability and quality of a power system. Considering the limitation of monitoring devices in distribution network, state estimation technique could be employed. State estimation is an action of appraising an unknown system variables based on limited number of realtime measurements. The main objective of this paper is to find the suitable state estimation that able to estimate the state of the islanded distribution network. In this paper, state estimation for a distribution network is developed and enhanced by incorporating composite load model. By incorporating this model, the calculated state variables of the network will be more accurate (to obtain the most practical value of the system state variables). Moreover, the proposed state estimation results are compared with backward-forward sweep load flow technique to justify the system state variables.
\end{abstract}

Index Terms-Distribution state Estimation, Distribution network, Load Modeling, Backward-forward sweep load flow.

\section{INTRODUCTION}

$\mathrm{O}$ NLINE monitoring in electrical power system is required for operation and control purposes such as load frequency control, voltage control and optimal dispatch power. Power generation, system frequency, voltages and power flows are common data that being monitored in power system network. This data is gathers at the control centre through Remote Terminal Units (RTUs) for real-time monitoring and used for operation and control purposes. An extensive real-time measurement would assist better control of the system. However in practice, measurement devices are very few due to high cost of these equipments. Only selected locations are being monitored, mainly at the critical area such as industrial zone and government administrative centre. The problem of limitation number of measurement equipment can be found in many conventional distribution systems. Very often distribution network is monitored only at the primary substation. Thus, to capture a perfect picture of the network operation seems impossible.

This work was supported by Malaysian Government and University of Malaya, Kuala Lumpur under HIR/MOHE research Grant (Grant code: D000004-16001)

Mazaher Karimi is with Department of Electrical Engineering, Faculty of Engineering, University of Malaya, Malaysia. (e-mail mazaher@siswa.um.edu.my).

Hazlie Mokhlis is with Department of Electrical Engineering, Faculty of Engineering, University of Malaya, Malaysia. (e-mail: hazli@um.edu.my).

Ab. Halim Abu Bakar is with UM Power Energy Dedicated Advanced Centre (UMPEDAC), Level 4, Wisma R\&D, University of Malaya, Malaysia. (e-mail: a.halim@um.edu.my)

Amidaddin Shahriari, Misagh Alaie Faradonbeh, Hazwani Mohd Rosli are with Department of Electrical Engineering, Faculty of Engineering, University of Malaya, Malaysia.
One way to address the limitation number of monitoring devices in determining the state condition of a distribution network is by using State Estimation (SE). SE has been introduced to power systems and implemented in steady state condition [1]. SE was implemented to power system in the late 1960's [1]. State estimator could approximate and estimate the power flows (active and reactive power) and voltages at a bus whose measurements are not available. An estimation of the state of a power system requires the measurement of electrical quantities, such as real and reactive power flows in transmission lines and real and reactive power injections at the buses [2]. SE is a data processing scheme to find the best possible values of the state. This implementation is still prevalent today. In the modern Energy Management System (EMS), SE program processes a set of raw measurement data and provides a real-time solution which is the basis of the advanced functions for system security monitoring and control [3].

$\mathrm{SE}$ is based on the mathematical relations between the system state variables and the measurements. The state estimator is used upon to process the available information and provides the best SE of the power system. The ability of the state estimator to achieve a high level of efficiency and numerical robustness is very importance in today's electric utility industry that heavily interconnected with loads. An algorithm must be converged from any starting point, and able to solve in practice both well-conditioned and illconditioned problems. It also should be able to address the limitation number of measurements in the system. One way of solving the limitation number of measurement is by using pseudo measurements. Customer load pseudo measurements are obtained from the historical data, load forecasting or other similar approximation methods in customer side. Hence, a few numbers of real-time measurements in a system is acceptable when using SE.

Various techniques have been used to obtain an SE solution, excellent surveys on SE algorithms can be found in [4-6]. Two types of data are required for SE; they are the network data and the measurement data. A real-time central control system can be used to improve the security and reliability of electrical power system [7]. In [8] is presented the integrated fuzzy SE and load flow analysis in distribution networks. A fusion method is applied in [9] in order to obtain a more reliable and accurate power system SE. A multi-area SE for distribution network is offered in [10], where own local SE is executed in each area of the network then, a minimum number of information of border will be shared to perform the SE of the whole network. A SE algorithm based on forward-backward propagation with higher $R / X$ ratio of lines has been presented on [11]. A branch estimation based on SE method, suitable for realtime monitoring of distribution network is presented in [12]. 
This paper presents enhancement of state estimation method by incorporating composite load model for distribution network application. The composite load model is incorporated to compute an accurate value of active and reactive power of various types of loads such as industrial, commercial and domestic. The proposed Distribution State Estimation (DSE) is based on Weighted Least Square (WLS) method to find and capture a network operation point. A minimum number of real-time measurements as in a practical distribution network are used in the proposed algorithm. Pseudo measurement is utilized in the proposed method to address the limitation number of measurements. The test result on two distribution networks show a significant improvement of the system state in term of overall voltage profiles.

\section{Problem Formulation of State Estimation}

A typical algorithm for SE uses the WLS algorithm that minimizes the sum of the squared weighted errors between the estimated and actual measurements. This method is popular among commercial state estimators. In this method, weights are selected as proportional to the accuracy of the measurements. Hence, measurement with high accuracy will has high weight.

In a power system including distribution and transmission system, the global measurement vector is indicated by $Z$. For an $N$-bus power system with $m$ measurements, assume $r$ an error vector to be standard Gaussian that has a zero mean and covariance $\sigma$. Let $i$ be the index of the measurements. The measurement equation is formulated in equation (1):

$$
Z_{i}=h_{i}(x)+r_{i}, \quad i=1,2, \ldots, m
$$

Where

$Z_{i}$ is the vector of measurements,

$X$ is the state vector,

$h_{i}(x)$ is the measurement matrix of the nonlinear function,

$r_{i}$ is the measurement errors vector.

Then, the measurement residual vector can be defined and formulated in equation (2) as follow:

$$
r_{i}=Z_{i}-h_{i}(x), \quad i=1,2, \ldots, m
$$

By applying the WLS method, it will minimize the objective function as describe in equation (3):

$$
J(x)=\sum_{i=1}^{m} \frac{\left(Z_{i}-h_{i}(x)\right)^{2}}{R_{i}}
$$

$R_{i}$ diagonal matrix of $\left[1 / \sigma_{1}{ }^{2}, 1 / \sigma_{2}{ }^{2}, \ldots, 1 / \sigma_{\mathrm{m}}{ }^{2}\right]$

$\sigma_{\mathrm{m}}{ }^{2}$ is the covariance of the $m^{\text {th }}$ measurement error.

The best solution of the state vector $X$ can be achieved by the solution of the following equation. It is calculated to reflect the expected accuracy of the corresponding meter used.

$$
[G(x)]\left[\Delta x^{k+1}\right]=\left[H\left(x^{k}\right)\right]^{T}[R]^{-1}\left[Z-h\left(x^{k}\right)\right]
$$

Where

$H(x)$ is the Jacobian matrix of the measurement function, $h(x)$, which is mentioned in equation (1).
$G(x)$ is called the Gain matrix; it can be defined and formulated in equation (5) as follow:

$$
[G(x)]=\left[H\left(x^{k}\right)\right]^{T}[R]^{-1}\left[H\left(x^{k}\right)\right]
$$

By solving the equation (4) to minimize such errors and find an optimal estimate, system state will be solved, iteratively. In this paper, WLS state estimation, as a mathematical analysis tools and an iterative solution, is presented to filter out such errors and find an optimal estimate of system states in distribution network.

The measurement matrix can be of a variety of most commonly used measurements in the practical system. The line power flows, bus power injections, bus voltage magnitudes and line current flows are common measurements in real power system. The proposed DSE is used the line power flows, bus power injections and bus voltage magnitudes in order to construct the measurement matrix.

Real and reactive power injection, $P_{i}$ and $Q_{i}$, equations at bus $i$ can be expressed by below:

$$
\begin{aligned}
& P_{i}=V_{i} \sum_{i=1}^{m}\left(G_{i j} \cos \theta_{i j}+B_{i j} \sin \theta_{i j}\right) V_{j} \\
& Q_{i}=V_{i} \sum_{i=1}^{m}\left(G_{i j} \sin \theta_{i j}-B_{i j} \cos \theta_{i j}\right) V_{j}
\end{aligned}
$$

Where

$G_{i j}$ is a line conductance from bus $i$ to bus $j$ in siemens (real part of admittance matrix),

$B_{i j}$ is a line susceptance from bus $i$ to bus $j$ in siemens (imaginary part of admittance matrix).

Real and reactive power flow, $P_{i j}$ and $Q_{i j}$, equations from bus $i$ to bus $j$ can be expressed as follow:

$$
\begin{gathered}
P_{i j}=V_{i}^{2}\left(G_{i j}+G_{i 0}\right)-V_{i} V_{j}\left(G_{i j} \cos \theta_{i j}+B_{i j} \sin \theta_{i j}\right) \\
Q_{i j}=-V_{i}^{2}\left(B_{i j}+B_{i 0}\right)-V_{i} V_{j}\left(G_{i j} \sin \theta_{i j}-B_{i j} \cos \theta_{i j}\right)
\end{gathered}
$$

The proposed DSE method is based on voltage measurements. The power injection and power flow is computed by using equations (6), (7), (8) and (9) at each buses of the distribution network. Finally, the calculated values are used to construct the measurement matrix $\left(h_{i}\right)$ in order to solve the Equation (4). Since real-time information is very limited in a conventional distribution system, the state estimator cannot attain convergence. Therefore, pseudo measurements are needed to assist convergence of the state estimator for distribution network. The pseudo measurements are used at some buses which measurement not available.

\section{INCORPORATING LOAD MODEL INTO STATE ESTIMATION}

In a real power system, load values are dependent on the voltage and system frequency. Loads are classified into different categories such as constant impedance, constant current and constant power. Hence, it is very crucial to include these models in SE to produce accurate estimation. For this reason, a composite load model that represents a mix type of load is used in this work. The model is incorporated into DSE by replacing the constant load power that usually used with composite load model based on the following equations: 


$$
\begin{gathered}
P_{i}=P_{i_{0}} *\left(C_{1}+C_{2}\left|V_{i}\right|+C_{3}\left|V_{i}\right|^{2}\right), \quad i=1,2, \ldots, m \\
Q_{i}=Q_{i_{0}} *\left(d_{1}+d_{2}\left|V_{i}\right|+d_{3}\left|V_{i}\right|^{2}\right), \quad i=1,2, \ldots, m
\end{gathered}
$$

Where,

$c_{1}$ and $d_{l}$ are constant power load compositions,

$c_{2}$ and $d_{2}$ are constant current load compositions,

$c_{3}$ and $d_{3}$ are constant impedance load compositions.

In these equations, $P_{i 0}$ and $Q_{i 0}$ is the nominal power at the rated voltage. However, when using this model, $P_{i 0}$ and $Q_{i 0}$ are normally taken as the values at initial system operating condition. In the proposed DSE, the load values are updated within iteration. Fig. 1 shows the line model of a radial distribution network connected with load $i$ and $j$ at both end of the line. The load value is calculated using equation (10) and (11).

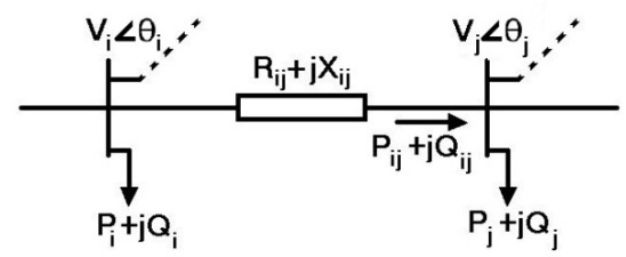

Fig.1. Model of line section of radial distribution system.

In this work, the constant variable of the composite load model is chosen according to the Table I.

TABLE I

THE VALUE OF THE COMPOSITE LOAD

\begin{tabular}{c|ccc}
\hline Load Type & $\mathrm{c} 1$ and $\mathrm{d} 1$ & $\mathrm{c} 2$ and $\mathrm{d} 2$ & $\mathrm{c} 3$ and $\mathrm{d} 3$ \\
\hline Active power & 0.4 & 0.3 & 0.3 \\
\hline Reactive power & 0.4 & 0.3 & 0.3 \\
\hline
\end{tabular}

\section{Simulation AND RESUlts}

The proposed DSE is tested on two radial distribution networks consisting of 12 buses of $11 \mathrm{kV}$ and 69 buses of $12.66 \mathrm{kV}$ systems. The proposed DSE uses the one measurement for voltage, active and reactive power flow at the main substation. For other buses, load data is from pseudo measurement, which obtained from historical data and the covariance of this type of measurement is assumed 0.25 .

\section{A. Case I}

The system configuration of 12 buses is shown in Fig. 2. The real-time monitoring is at bus number 1. Line data and nominal load data is given in Table II.

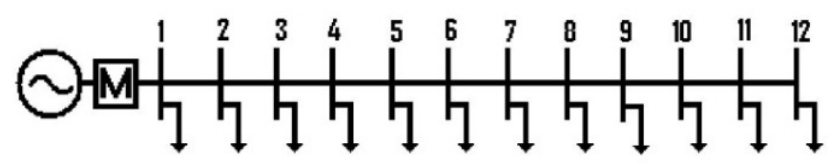

Fig.2. Test system configuration for case I

The magnitude of voltages and angles of each bus are shown in Fig. 3 and Fig. 4, respectively. In each figure, three results are presented; DSE with and without load model and load flow with load model. The load flow is based on backward-forward sweep method. From Fig. 3, it can be observed that voltage profile of the DSE with load modelling produces voltage profile close to the load flow voltage profile. Similarly, the phase angle profile also shows the same pattern as in the voltage profile. It can also be observed that the system state accuracy is decreasing as the bus distance moving far from the measurement point.

TABLE II

THE PARAMETERS OF THE LINES AND LOAD VALUES

\begin{tabular}{c|c|cccc}
\hline $\begin{array}{c}\text { Send } \\
\text { Bus }\end{array}$ & $\begin{array}{c}\text { Receive } \\
\text { Bus }\end{array}$ & $\mathrm{R}(\mathrm{pu})$ & $\mathrm{X}(\mathrm{pu})$ & $\mathrm{P}(\mathrm{MW})$ & $\mathrm{Q}(\mathrm{MVar})$ \\
\hline 1 & 2 & 0.00009 & 0.000038 & 0.06 & 0.06 \\
\hline 2 & 3 & 0.000098 & 0.000041 & 0.04 & 0.03 \\
\hline 3 & 4 & 0.000173 & 0.000072 & 0.055 & 0.055 \\
\hline 4 & 5 & 0.000263 & 0.00011 & 0.03 & 0.03 \\
\hline 5 & 6 & 0.00009 & 0.000038 & 0.02 & 0.015 \\
\hline 6 & 7 & 0.000083 & 0.000034 & 0.055 & 0.055 \\
\hline 7 & 8 & 0.000364 & 0.0001 & 0.045 & 0.045 \\
\hline 8 & 9 & 0.000466 & 0.000132 & 0.04 & 0.04 \\
\hline 9 & 10 & 0.000239 & 0.000068 & 0.035 & 0.03 \\
\hline 10 & 11 & 0.000125 & 0.000035 & 0.04 & 0.03 \\
\hline 11 & 12 & 0.000102 & 0.000029 & 0.015 & 0.015 \\
\hline
\end{tabular}

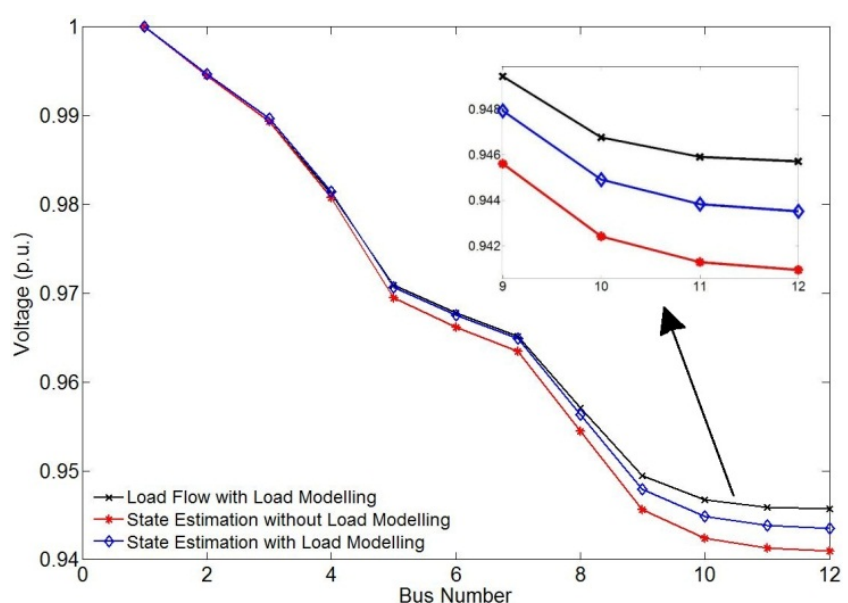

Fig.3. Voltage amplitude in case I

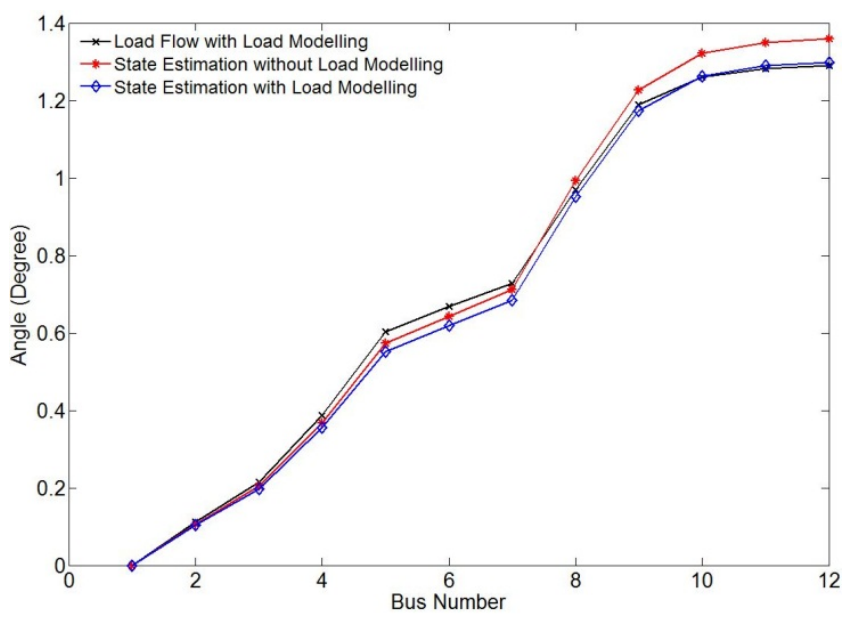

Fig.4. Bus angle curve in case I

TABLE $I I I$

ERROR VALUE OF VOLTAGES IN CASE I

\begin{tabular}{c|cccc}
\hline \multirow{2}{*}{$\begin{array}{c}\text { Bus } \\
\text { No. }\end{array}$} & \multicolumn{2}{|c}{ State Estimation } & $\begin{array}{c}\text { Load Flow } \\
\text { with Load }\end{array}$ & Error \% \\
& Modelling & $\begin{array}{c}\text { with Load } \\
\text { Modelling }\end{array}$ & $\begin{array}{c}\text { Modelling } \\
\text { Moun Load }\end{array}$ & \\
\hline 1 & 1.0 & 1.0 & 1.0 & 0.0000 \\
2 & 0.994490 & 0.994669 & 0.994503 & -0.0167 \\
3 & 0.989346 & 0.989716 & 0.989371 & -0.0349 \\
4 & 0.980781 & 0.981482 & 0.981240 & -0.0246 \\
5 & 0.969522 & 0.970696 & 0.970933 & 0.0244 \\
6 & 0.966206 & 0.967526 & 0.967784 & 0.0267 \\
7 & 0.963467 & 0.964912 & 0.965123 & 0.0219 \\
8 & 0.954465 & 0.956348 & 0.957083 & 0.0768 \\
9 & 0.945603 & 0.947938 & 0.949447 & 0.1589 \\
10 & 0.942413 & 0.944914 & 0.946768 & 0.1958 \\
11 & 0.941279 & 0.943839 & 0.945917 & 0.2197 \\
12 & 0.940946 & 0.943524 & 0.945719 & 0.2322 \\
\hline
\end{tabular}




\section{B. Case II}

In this case, the network configuration is shown in Fig.5. The complete line data and nominal load data is given in [13]. In this test case, measurement of voltage and power flow is taken at main substation in the network. Meanwhile, 6 measurements of injected power are taken at bus $8,11,28$, 36,50 and 53. For other buses, pseudo measurement is applied.

The amplitude of the voltage and angle values in all buses is presented in Fig. 6 and Fig. 7 respectively. Similar pattern can be observed as in the case I, where voltage and phase angle profile of the DSE with load modelling is close to the voltage profile of the load flow. The result in Fig. 7 shows that the voltage magnitude is close to each other for buses where measurement is taken. This pattern can be seen at bus 8,11 and 53. By comparing the SE with and without load modelling to load flow at bus 61 , it can be found that $1.4972 \%$ of improvement has been achieved, which is a significant improvement as compared without load model in SE.

Both test cases indicated that the proposed DSE method with load modelling improve significantly the estimated voltage and angle to be close as in load flow with load modelling.

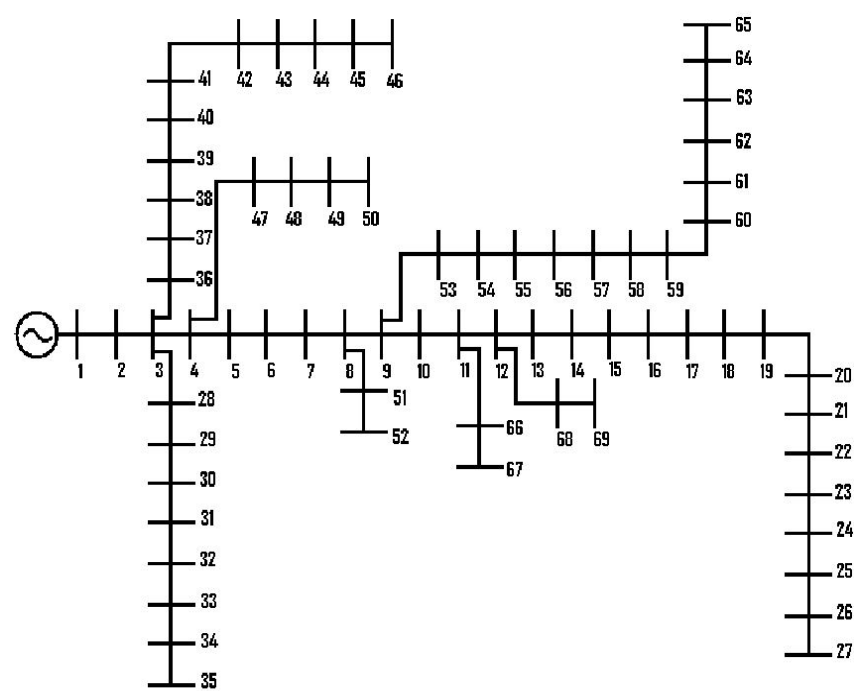

Fig.5. Test system configuration for case II

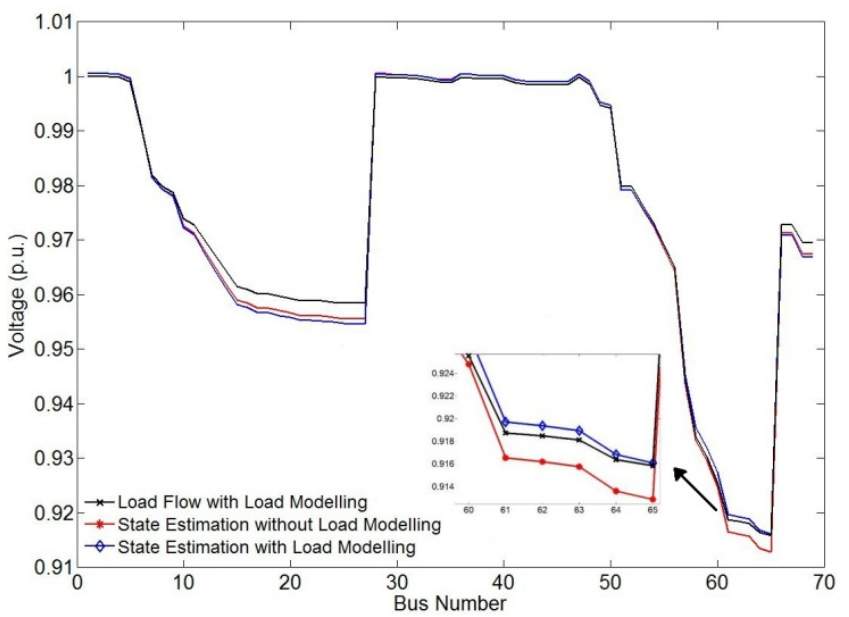

Fig.6. Voltage amplitude in case II

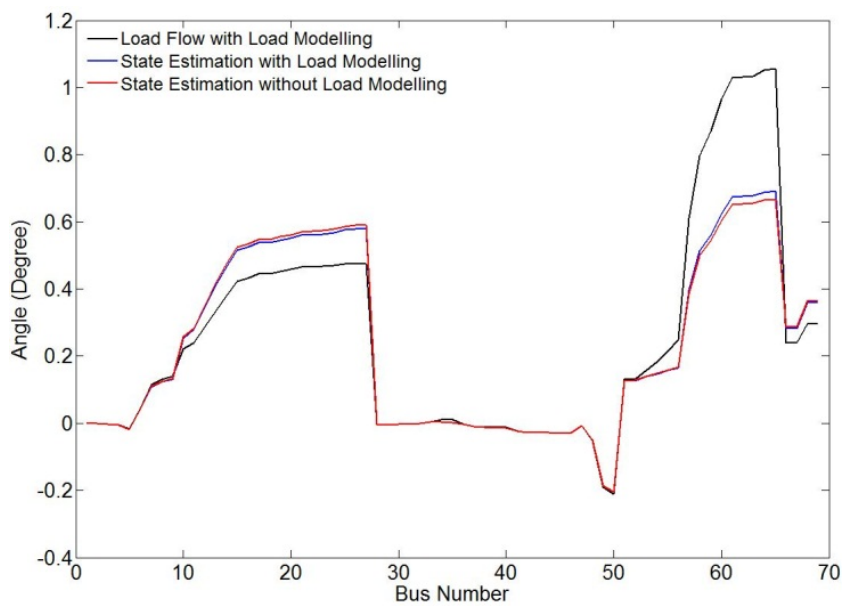

Fig.7. Bus angle curve in case II

\section{CONCLUSION}

This paper proposes an improvement of SE method for distribution system that equipped with minimum number of measurements. The DSE has been incorporated with a composite load model. By having this load model, any type of load in distribution system can be represented and therefore the distribution state estimation result would be more accurate.

The test results on 12 and 69 bus systems have proven that the voltage profiles have been improved significantly by $0.5047 \%$ and $1.4972 \%$, respectively. The test result also shows the robustness of the method since it can solve DSE problem with minimum number of measurement. This is an important feature to determine state variable of a distribution system that has minimum number of measurements.

\section{ACKNOWLEDGEMENT}

This work was supported by Malaysian Government and University of Malaya, Kuala Lumpur under HIR/MOHE research Grant (Grant code: D000004-16001).

\section{REFERENCES}

B. Fardanesh and S. Zelingher, Power system state estimation: modeling error effects and impact on system operation, (2001)

[2] T. Nagsarkar and M. Sukhija, Power System Analysis: Oxford University Press, (2007)

[3] C. Lu, et al., Distribution system state estimation, IEEE Trans. Power Systems, vol. 10(1995), pp. 229-240

[4] F. F. Wu, Power system state estimation: a survey, International Journal of Electrical Power \& Energy Systems, vol. 12(1990), pp. 80-87

[5] A. da Silva and D. Falcao, Bibliography on power system state estimation (1968-1989),IEEE Trans. Power Systems, vol. 5(1990), pp. 950-961

[6] A. Bose and K. A. Clements, Real-time modeling of power networks, Proceedings of the IEEE, vol. 75(1987), pp. 1607-1622

[7] F. C. Schweppe, Power system static-state estimation, Part III: Implementation, IEEE Trans. Power Apparatus and Systems, (1970), pp. 130-135 A. T. Saric and R. M. Ciric, Integrated fuzzy state estimation and load flow analysis in distribution 
networks, IEEE Trans. Power Delivery, vol. 18(2003), pp. 571-578

[9] N. V. Nguyen, et al., Power system state estimation with fusion method, (2010), pp. 71-76.

[10] G. N. Korres, A distributed multiarea state estimation, IEEE Trans. Power Systems, (2011), pp. 1-12

[11] D. Thukaram, et al., A robust three-phase state estimation algorithm for distribution networks, Electric Power Systems Research, vol. 55 (2000), pp. 191-200

[12] Y. Deng, et al., A branch-estimation-based state estimation method for radial distribution systems, IEEE Trans. Power Delivery, vol. 17 (2002), pp. 1057-1062

[13] M. Baran and F. F. Wu, Optimal sizing of capacitors placed on a radial distribution system, IEEE Trans. Power Delivery, vol. 4(1989), pp.735743 